\title{
LA PHYLLOTAXIE DU BANANIER et ses conséquences pour la compréhension du système rejetonnant
}

\author{
par E. DE LANGHE
}

Ingénieur Agronome (Gand) ( $\left.{ }^{1}\right)$

\begin{abstract}
La culture intensive du bananier est inévitablement liée aux problèmes du pouvoir rejetonnant. Bien que le cultivateur soit conduit à essayer d'adapter d'une façon empirique le rejetonnage ou émission des rejets aux conditions écologiques locales, il serait autrement avantageux de pouvoir fixer d'abord des règles générales. Par la suite, et pour chaque milieu écologique, des règles spéciales pourraient être concues.

Ces derniers temps, plusieurs travaux de recherches ont été consacrés spécialement à la nature exacte de l'émission des rejets et aux facteurs pouvant l'activer ou l'inhiber $\left({ }^{2}\right)$.

Le Centre de Recherches de l'I. N.E.A. C. (Institut National pour l'Étude A gronomique au Congo) s'était attaché depuis plusieurs années au problème de l'amélioration du bananier plantain. Dès le début, le pouvoir rejetonnant ainsi que ses variations spécifiques ou écologiques ont attivé l'attention des chercheurs. Aussi sommes-nous reconnaissant à la rédaction de "Fruits" d'avoir bien voulu publier quelques résultats de ces recherches.
\end{abstract}

\section{PHYLLOTAXIE}

\section{A. Constatations.}

Généralement on admet que les feuilles du bananier se trouvent sur trois rangées en une seule spirale tournée vers la gauche (Kervegant, I935). Au premier coup d'œil cependant les feuilles, ou plutôt les gaines foliaires, semblent s'imbriquer en deux hélices presque opposées et tournées vers la droite. Cette impression incite Dumas (I955) à supposer que le point végétatif comporterait deux centres générateurs de feuilles en accord avec la théorie Plantefol.

Dans le "Pfanzenreich », K. Schuman (Igoo) parle d'un angle de divergence égal à $3 / 7$. Sкuтch (Ig27, I932) remarque une variation de cet angle qui passe de $\mathrm{I} / 3$ chez les rejetons via $2 / 5$ à $3 / 7$ chez les grands rejets, à $4 / 9$ chez les plantes adultes. Et Simmonds (I959) ajoute qu'en somme l'angle varie donc entre $\mathrm{I} 2 \mathrm{O}^{\circ}$ et $16 \mathrm{O}^{\circ}$.

Ceci représente tout ce qu'on trouve dans la littérature concernant la phyllotaxie du bananier.

Selon Simmonds, l'angle augmente d'une façon continue à partir du stade très jeune du rejeton. Or, l'inflorescence contient un nombre considérable de glomérules (excédant de 3 à 6 fois le nombre de nœuds

(I) Attaché de recherches à l'IBERSOM, ancien assistant à l'INEAC, Yangambi.

(2) N.D. L. R. L'Institut Français de Recherches Fruitières (I. F. A. C.) a déjà publié dans cette revue le résultat d'observations préliminaires effectuées sur des bananiers du groupe "Sinensis ". (Voir bibliographie). sur la partie végétative). Ou bien les glomérules ne sont pas des nœuds véritables et leur angle de divergence obéit à d'autres lois. Ou bien les glomérules sont des nœuds, et l'angle de divergence doit de nouveau diminuer à un certain stade, car, au rythme supposé par Simmonds (de I20 à I6o degrés en quelque soixante nœuds), l'angle de divergence atteindrait des valeurs invraisemblables pour l'extrémité de l'inflorescence.

I. Les noeuds de l'inflorescence, c'est-à-dire les glomérules, sont-ils identiques aux nouds foliaires, sur le plan anatomique? Ou encore : les bractées correspondent-elles bien à des feuilles métamorphosées ?

$\mathrm{Nul}$ observateur régulier du bananier ne doute de cette dernière évidence, car il suffit de suivre l'évolution structurale à travers les dernières feuilles émises avant l'inflorescence pour constater que le pétiole et la gaine foliaire s'élargissent, et que le limbe diminue ; le " pavillon " aura fréquemment l'aspect d'une bractée encore verte pourvue de deux petits limbes; suivent deux bractées stériles (ne contenant pas de fleurs) dont la première est intermédiaire entre le " pavillon " et la seconde bractée.

Ajoutons cependant encore quelques arguments de poids :

- le genre Ensete (de la même sous-famille des Musoïdeae) connaît une transformation vraiment continue entre les feuilles et les bractées au cours de la floraison.

- dans le genre Eumusa, Musa acuminata ssp. bur- 


\section{Lu dans Fruits, il y a 50 ans ...}

manica montre, au bout des bractées de l'inflorescence femelle, des traces très nettes d'un limbe vert.

- Deux cas tératologiques :

a. Lorsque la variété $I$ bota (probablement un AAAafricain) est attaquée par la maladie du BunchyTop, pendant la floraison, ses bractées commencent à développer un limbe minuscule à l'apex, et se décolorent partiellement.

b. L'étrange Libanga (une variété du Plantain Cornu, appelée Ebanga ou Gibanga au Gabon, WALKer, I93I) possède une inflorescence mâle fort rudimentaire, d'une trentaine de mains, qui s'arrête sur une fleur terminale. Les dernières bractées possèdent des limbes minces, et même le périanthe méconnaissable de la fleur terminale semble se composer de bractées rudimentaires pourvues de limbes verts (fig. I).

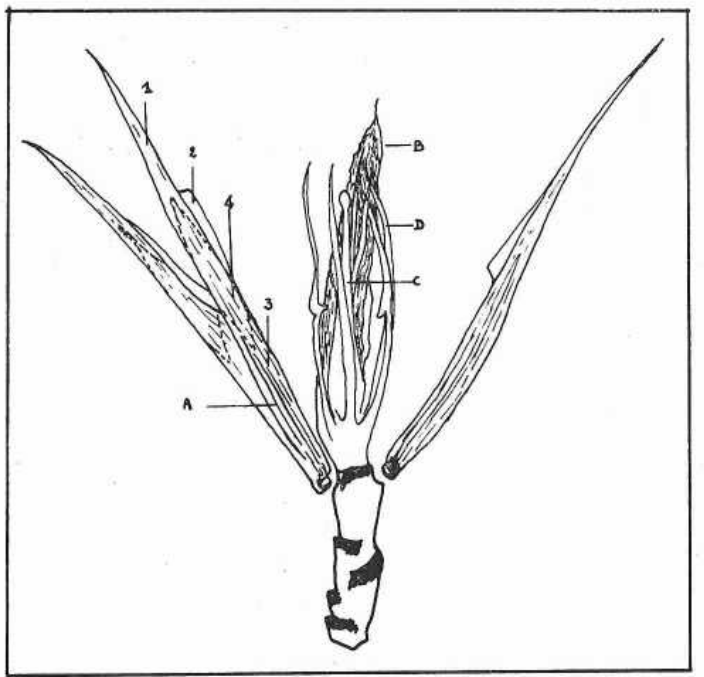

FIG. I. - Fleur terminale d'une variété plantain Libanga.

A. Deux tépales soudés bractéiformes, - B. Tépale libre (?) C. Style. - D. Étamines.

x. Blanc, -2 . Transparent. - 3. Rouge. -4 . Vert léger.

Les glomérules sont donc de véritables nœuds.

2. Tous les nouds d'un bananier sont effectivement posés en une seule spirale, et, comme on le verra plus loin, l'angle de divergence sur l'inflorescence varie entre I25 et I30 degrés. Il diminue donc effectivement après la partie foliaire pure.

En conséquence, la phyllotaxie ne semble pas si simple ; elle n'augmente certainement pas d'une façon continue.

\section{B. Observations.}

I. La spirale unique est clairement mise en évidence par l'inflorescence d'une variété de plantains, bien connue en Afrique occidentale sous le nom de Mpizina (WALKer, I93I) (Aleke, Muller, I947). Tous les nceuds (glomérules) se sont soudés en une seule spirale, portant une seule bractée spiraloïde qui se déchire en lambeaux pendant son développement final (fig. 2).

Le même phénomène s'observe chez la variété Ulongan du groupe AA de la Nouvelle-Guinée (SIMMONDS, I959).

2. L'angle de divergence et son évolution : nous l'avons étudié chez deux variétés au Congo: Afati (un plantain commun $\mathrm{AAB}$ avec nervures et gaines foliaires rougeâtres) et Nyira-Bahema (un AAA-africain) *.

Nous discernons les stades (ou zones) suivants :

a. Rejetons : formés d'écailles plus ou moins allongées mais sans aucune trace de limbe. Les rejetons dépassent rarement une hauteur de $30 \mathrm{~cm}$.

b. Rejet épée (sword sucker) : les écailles portent des limbes lancéiformes. Ces rejets dépassent parfois par leur vigueur la hauteur de I $\mathrm{m}$.

c. Rejet adulte I : le rejet a produit au moins Io feuilles normales.

d. Rejet adulte 2 : le rejet a produit au moins 25 feuilles normales.

e. Porteur d'inflorescence femelle: tandis que les dernières feuilles sont développées, l'inflorescence est sortie.

f. Inflorescence femelle.

g. Inflorescence mâle I : moins de 30 glomérules.

h. Inflorescence mâle 2 : de 50 à roo glomérules et plus.

Cinq échantillons de chaque stade ont été analysés de la façon suivante :

- un disque d'une certaine hauteur $(20 \mathrm{~cm}$ au moins) est enlevé à mi-hauteur entre le tubercule et la base du pétiole correspondant à la dernière feuille déroulée (ou simplement émergée s'il s'agit d'une feuille de rejet épée) ;

- une alidade transparente est posée sur la coupe transversale bien horizontale, avec son centre ajusté sur la plus jeune feuille encore enroulée au milieu ;

- l'angle de divergence entre deux feuilles successives est matérialisé par les deux lignes qui relient le

* Les symboles A ou B désignent des génomes du types Musa acuminata et Musa balbisiana, et leurs combinaisons sont employées conformément à l'hypothèse de travail de Simmonds et SHEPHERD (1955). 


\section{Lu dans Fruits, il y a 50 ans ...}

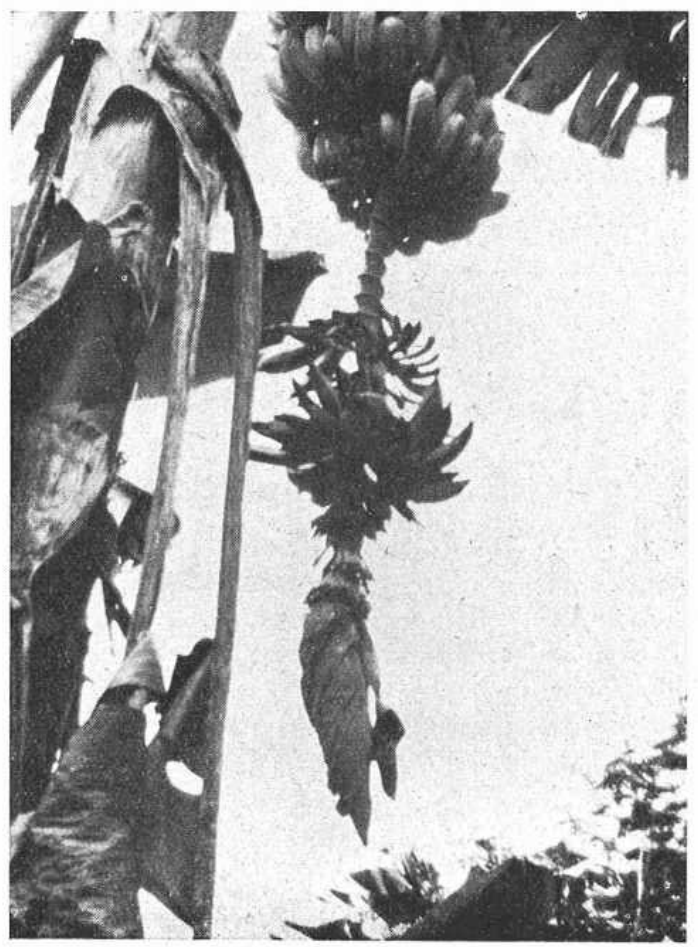

centre aux points où se rejoignent (éventuellement se rapprochent) les bords de la gaine foliaire. Nous préférons ce point au point opposé, c'est-à-dire le milieu de la gaine, à cause de l'effet de nervure qui commence déjà à ce niveau, et qui s'exprime au maximum sur la partie découverte de la gaine. En effet, à cet endroit, la gaine n'est pas arrondie, mais accuse deux angles dorsaux dont celui de gauche est plus développé, ce qui donne à la coupe transversale une allure asymétrique ;

- les nœuds des dernières feuilles et des bractées stériles sont comparés d'une autre façon : toutes les feuilles sont enlevées y compris les dernières, ainsi que les bractées stériles. Il reste la tige centrale, se terminant via la crosse en une hampe florale. Quelques nœuds foliaires sont nettement visibles le long de la tige ; ils la contournent et leurs deux extrémités se rejoignent en un angle aigu pointé vers le bas. Cette pointe est maintenant prise comme point de repère; il est facile de la situer (à l'aide d'une règle) au niveau du nœud inférieur. La tige est coupée transversalement juste au-dessus de ce nœud, et l'alidade permet ainsi de voir l'angle de divergence entre les deux nœuds en
Fig. 2. - Inflorescence en spiraloïde chez Musa paradisiaca, cultivar: Aleke. Les fleurs sont attachées à un seul glomérule ininterrompu. La fructification de quelques fleurs soi-disant neutres est un phénomène relativement fréquent chez cette variété.

question. Cette opération est répétée de haut en bas, depuis la crosse jusqu'au rhizome ;

- quant à la crosse même, la seule façon de transposer les points de repère est de suivre les légères cannelures qui se dessinent parallèlement sur son contour;

- sur l'axe floral, le milieu des glomérules est facile à repérer et l'alidade, mise sous un tronçon, permettra de mesurer une série d'angles de divergence en une seule fois;

- l'extrémité de l'inflorescence mâle est analysée par une simple coupe transversale du bouton terminal vers la fin de la fructification. L'angle sera matérialisé par le centre du bouton et les points médians de deux bractées successives ;

- différents types d'angle ont été observés : entre des feuilles successives $I, 2,3,4 \ldots$; entre les feuilles I, $3,5,7 \ldots$ ou encore entre les feuilles $I, 4,7 \ldots$, dans le but de discerner éventuellement plusieurs spirales très régulières et donc plusieurs centres de formation foliaires dans le tubercule. Il s'avérait que seul le premier type est à retenir, les autres types ne pouvant parfois compter que pour une seule des zones.

Remarque: Pour les quatre zones a, b, c, d, nous avons fait appel à autant de pseudo-troncs différents, tandis que les zones e, $\mathrm{f}, \mathrm{g}, \mathrm{h}$, furent obsiervées sur un même échantillon.

3. Sous-développement: quelques observations ont été effectuées sur des rejets sous-développés (watersuckers), ainsi que sur des "porteurs d'inflorescence" sous-développés.

\section{Résultats.}

Vu le caractère non systématique de ce genre d'observations (voir rubrique B 2, Remarque) l'analyse statistique des données est impossible. Nous nous sommes limité, par conséquent, à décrire l'allure générale que prennent ces angles de divergence.

I. Les valeurs (en degrés) entre lesquelles l'angle varie dans chaque zone ;

2. A l'intérieur des zones $(\mathrm{a}+\mathrm{b})(\mathrm{c}+\mathrm{d})$ une évolution dans l'angle n'a pas été relevée.

Une évolution rapide se remarque dans la zone "e ", une évolution lente probablement chez Nyira-Bahema dans la zone $(g+h)$. Il existe par contre une transition brusque de l'angle, aux passages (b dans c) (e dans f). 


\section{Lu dans Fruits, il y a 50 ans ...}

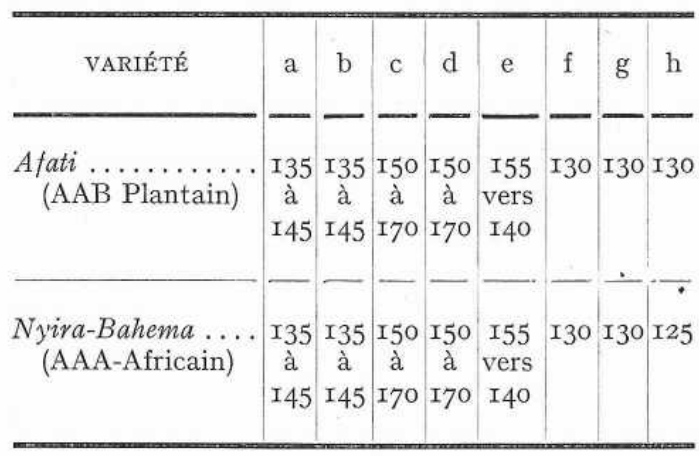

3. Cependant, si l'angle n'évolue pas dans la plupart des zones, il n'en est pas pour autant stabilisé. A l'intérieur de la zone " d ", l'étude de la distribution des 50 mesures ( 5 échantillons $\times$ II feuilles caractéristiques de cette zone) fait ressortir deux maxima de fréquence, situés vers $I_{55}$ et vers ${ }^{6} 65$ degrés (fig. 3). Ces deux types d'angle alternent sur une même série de feuilles.

Un pied Afati montre par exemple successivement les angles suivants : $I 64, I 56, I 63, I 56, I 59, I 54, I 65$, I56, I62, I53, I64, I68, I55, I56, I65, I59. Cette alternance n'est donc pas une règle générale. Nous ne trouvons pas d'explication logique pour ce phénomène remarquable.
4. Chez les rejets sous-développés; où les feuilles portaient déjà des limbes foliaires (water-suckers), l'angle de divergence atteignait une valeur de $r_{50}$ à I6o degrés.

5. Des points 2 et 4 , on déduit que l'apparition des limbes foliaires semble être en relation avec l'amplification de l'angle de divergence.

6. Il est connu qu'un pied sous-développé tarde à fleurir et produit beaucoup plus de feuilles qu'un pied normal d'une même variété. Nous avons remarqué que les angles de divergences dépassent dans ce cas fréquemment les r7o degrés.

Dans la figure $\mathrm{n}^{\circ} 4$, l'évolution de l'angle est portée en relation avec le nombre de nœuds développés. Le sous-développement est schématisé par une ligne pointillée.

La figure $n^{\circ} 5$ montre finalement le diagramme simplifié de la phyllotaxie telle que nous la concevons. Un nombre très restreint de nœuds posés sur une spirale unique représente chaque stade. En partant de l'extérieur, nous trouvons successivement les stades $(\mathrm{a}+\mathrm{b}=$ rejet jeune), $(\mathrm{c}+\mathrm{d}=$ rejet adulte $=$ branche); e (stade intermédiaire), $\mathrm{f}+\mathrm{g}+\mathrm{h}=$ inflorescence).

La ligne pleine, reliant les nœuds de $(c+d)$ montre comment la disposition des feuilles adultes fait penser à deux spirales, tournant vers la droite.

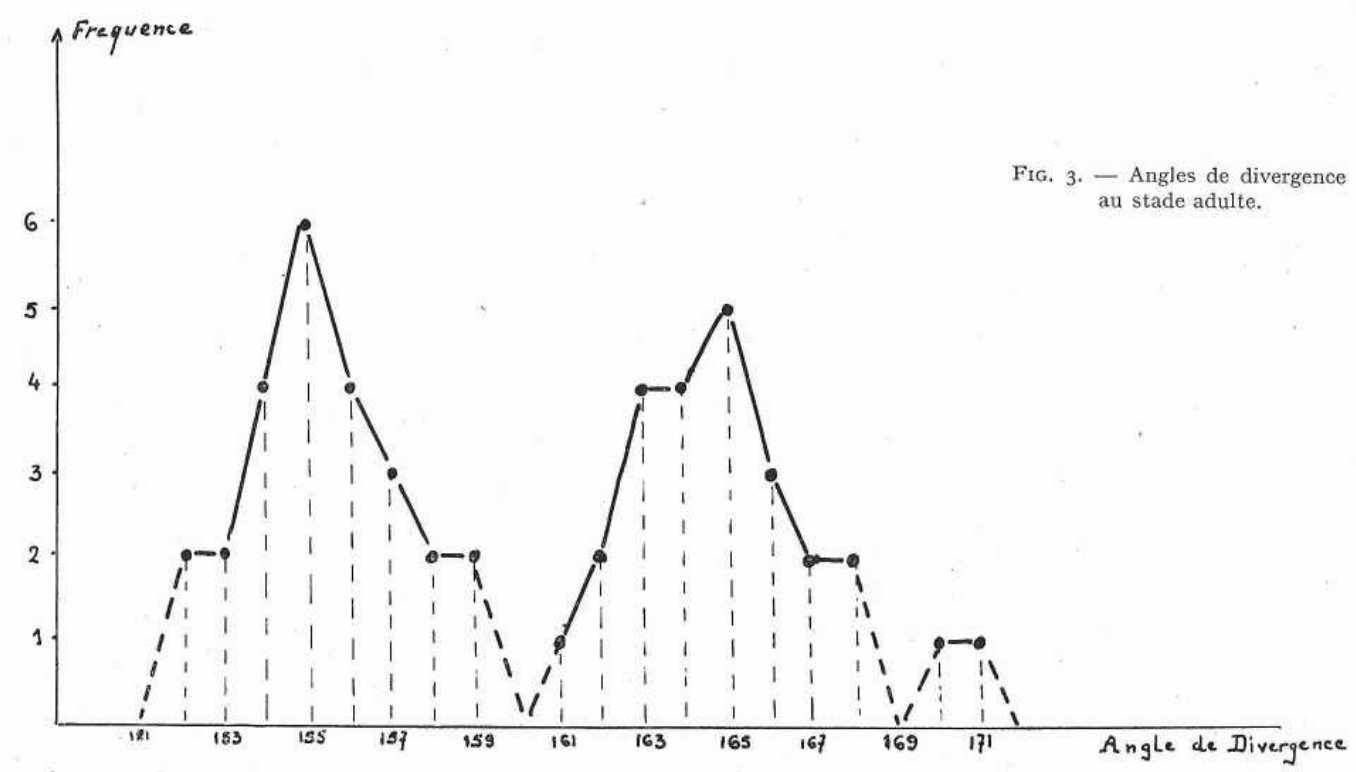




\section{Lu dans Fruits, il y a 50 ans ...}

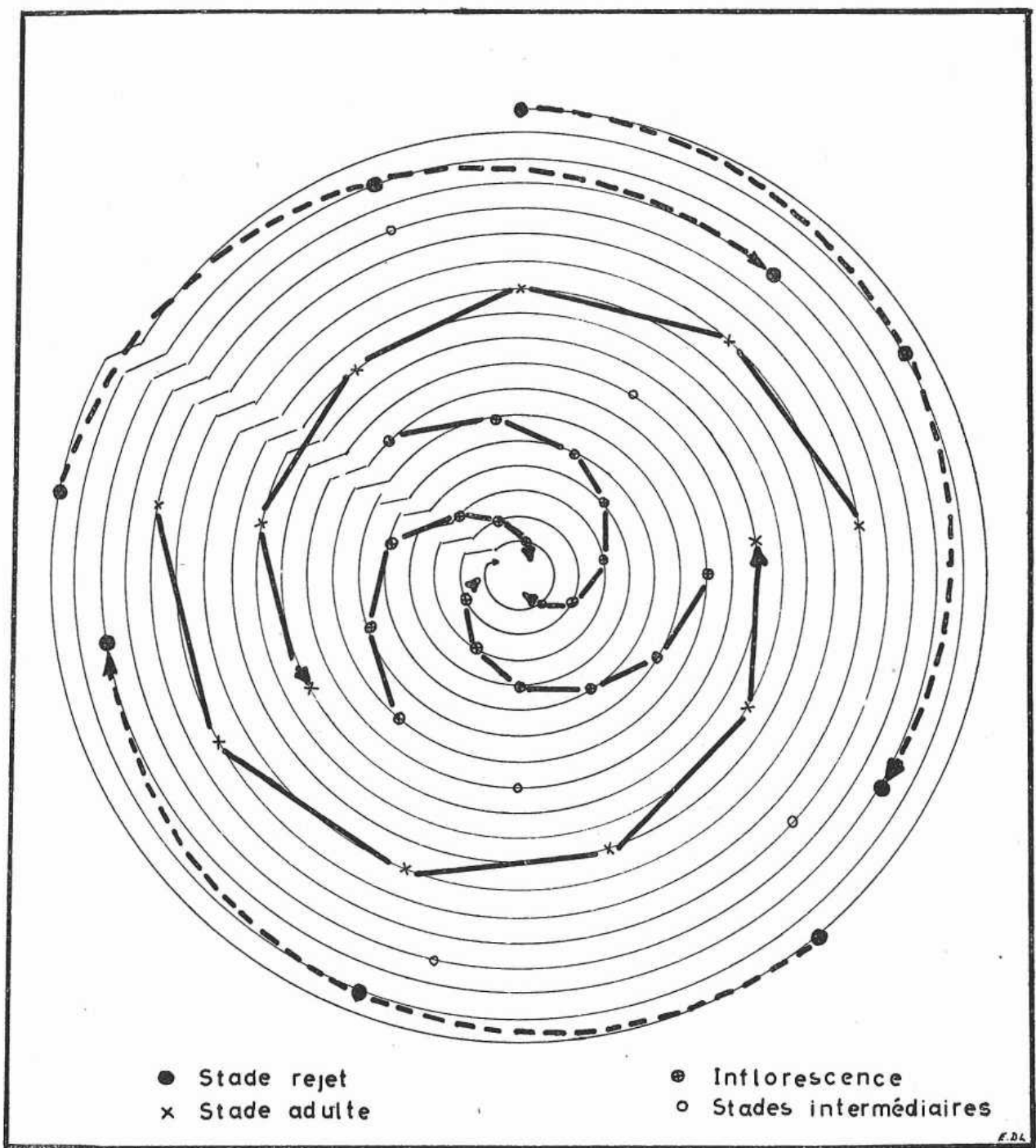

FIG. 5. - Phyllotaxie du bananier.

\section{MORPHOLOGIE DU REJETONNAGE}

\section{A. Constatations.}

Selon Skutch, un rejet prend naissance sur le tubercule, du côté opposé de chaque feuille et dans l'angle formé par les deux bords de la gaine foliaire qui se rejoignent à sa base en forme de " V ». L'idée est reprise par BARKER (I959) qui l'exploite pour en déduire un système original de multiplication végétative.

Or, chaque rejet n'est autre qu'une tige dont les premiers nœuds sont fort rapprochés, mais dont l'in- 


\section{Lu dans Fruits, il y a 50 ans ...}

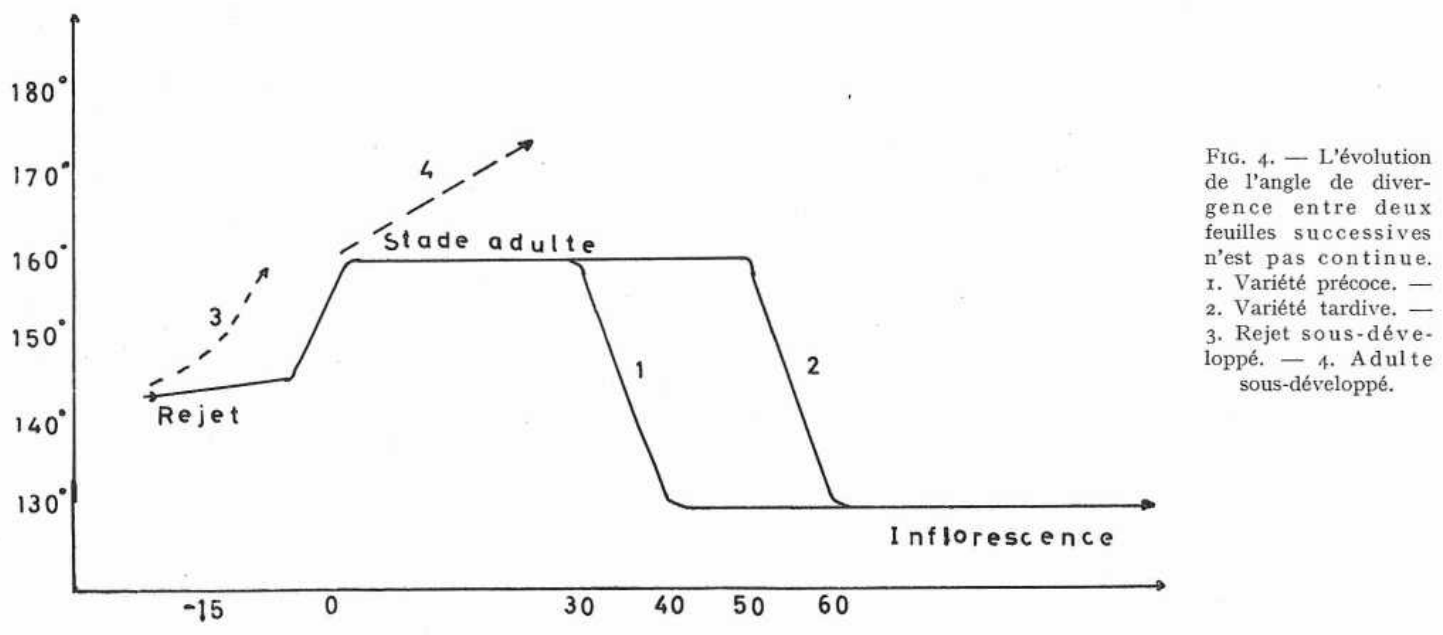

florescence terminale est portée par une hampe florale qui a traversé le pseudo-tronc formé par les feuilles imbriquées. En admettant que cette tige soit opposée à la feuille, par rapport à l'axe central (c'est-à-dire le tubercule) elle ne peut être considérée comme une branche, car les branches naissent à l'aisselle même des feuilles. La véritable branche serait l'axe "central ". Et SKUTCH propose en effet une structure sympodiale pour le rhizome.

La figure $6 \mathrm{~A}$ représente cette structure. Les morceaux verticaux des branches successives forment un pseudo-axe central, qui devient tubercule. Les bouts des tiges forment les " rejets".

\section{B. Observations.}

L'hypothèse de la structure sympodiale entraîne une complication pour les points suivants :

I. Les feuilles $f$ I, f 2, etc. (fig. 6 A) devraient par leur réseau vasculaire être visiblement liées aux rejets R I, R 2. Or, nulle part, même pas chez les plus jeunes bourgeons en formation, on ne remarque ce lien. La feuille en question s'implante donc sur le pseudo-axe central et nullement sur le rejet.

L'idée du sympodium n'est soutenable qu'en présupposant la résorption d'une partie du tissu conducteur initial des rejets par la couche dictyogène de Mangin, c'est-à-dire le cylindre central du tubercule dont les cellules se gorgent de grains d'amidon.

2. Les dernières feuilles sont portées par l'axe floral qui a traversé le pseudo-tronc. On ne relève aucune trace de bourgeons aux nœuds de ces dernières feuilles.
Or, ces feuilles s'implantent exactement de la même manière et avec au début les mêmes angles de divergence que les " feuilles à bourgeon opposé ». Ici, ainsi que pendant toute la floraison, le bananier semble être un vrai monopodium.

A moins que de nouveau, on admette une atrophie précoce du primordium des bourgeons.

3. Au début de son développement, le rejet ne forme que des feuilles, et ne montre aucune trace de rejetonnage. Il faut donc admettre qu'ici également la plante se comporte comme un monopodium parfait.

4. Par ailleurs, ce rejetonnage remet en question le schéma de la structure sympodiale. Chaque rejet (R I, R 2, etc.) s'il se développe normalement émet à un certain moment à son tour un bourgeon presque toujours du côté opposé au pseudo-axe central (SuBRA et GUILlfmot, I96I). Ces nouveaux rejets ne peuvent être, selon l'hypothèse discutée, que le prolongement des branches B I, B 2, etc. La figure 6 B représente le schéma complété et compliqué du sympodium chez le bananier. En fait, le bout de la branche B I, au lieu d'arrêter son développement (fig. 6 A) ne cesse de former de nouvelles branches, et sa structure nous rappelle singulièrement le monopodium.

5. Dans la famille des Musacées, les Strelitzoïdeae dont l'angle de divergence est égal à $180^{\circ}$ (ou I/2) ont une structure monopodiale; tandis que les Musoïdeae, dont l'angle de divergence tend vers cette valeur, auraient une structure sympodiale. Une différence morphologique si fondamentale dans une même famille est unique. Si elle se manifestait clairement dans la morphologie florale, on serait peut-être enclin à distan- 


\section{Lu dans Fruits, il y a 50 ans ...}

cer les deux groupes au point de vue systématique. Mais les deux types floraux montrent la même structure monopodiale !

\section{La structure monopodiale du bananier.}

Tout le problème se cristallise autour de la question de savoir si le bourgeon opposé à une feuille prend naissance à partir d'un tissu commun, ou s'il montre des points communs avec une autre feuille, par exemple celle qui le recouvre. La reproduction d'une coupe faite à l'endroit d'un bourgeon en développement (fig. 7) démontre clairement au moins une évidence : à l'endroit du bourgeon $\mathrm{A}$, les deux bords de la feuille opposée $\mathrm{C}$, ne sont plus que des pellicules minces qui se soudent avec l'épiderme reliant les feuilles B et D. Aucune trace de tissu conducteur pour la feuille C, et le bourgeon $\mathrm{A}$ en est entièrement indépendant.

Par contre, la zone corticale située entre le bourgeon et le réseau vasculaire intérieur de la feuille $B$, est traversée par des vaisseaux arqués qui semblent relier les deux organes. Nous reconnaissons cependant que l'exemple n'est pas assez démonstratif.

Mais il existe un autre critère. Dans un sympodium (fig. 6) le bout de chaque tige $\mathrm{R}_{1}, \mathrm{R}_{2} \ldots$... doit se développer simultanément avec la feuille correspondante (f $I, f 2 \ldots$...). Autrement dit, l'hypothèse de la structure sympodiale n'admet pas le développement d'un bourgeon après celui de la feuille opposée.

Or, les coupes effectuées sur les plus jeunes organes de plusieurs bananiers, n'ont jamais montré une trace de bourgeon opposé à une feuille qui s'allonge déjà à l'intérieur du pseudo-tronc (et qui est par conséquent déjà complètement formée). La figure 8 montre une coupe radiale observée sur un primordium du Bananier Nain. (Dwarf Cavendish). Par ailleurs, on remarque suffisamment sur la figure 7 le décalage de développement existant entre le bourgeon A et la feuille B. Nous appuyons sur le fait que ces feuilles (B, D et même les suivantes) sont déjà entièrement sorties du pseudotronc. Les bourgeons naissent et se développent bien après la formation des fewilles correspondantes.

Ici donc, l'hypothèse du sympodium ne nous semble plus défendable.

Par contre, la structure monopodiale permet de considérer le cylindre central comme une véritable tige centrale, et les rejets comme des branches (fig. 6 C). Ces rejets se forment longtemps après le développement des ferilles qui les recouvrent. Les points qui compliquèrent l'hypothèse du sympodium s'intègrent parfaitement dans l'idée du monopodium :

FIG. 6. - Les structures possibles du bananier.

A. Schéma d'un sympodium en général. - B. La structure sympodiale du bananier. - C. La structure monopodiale du bananier.

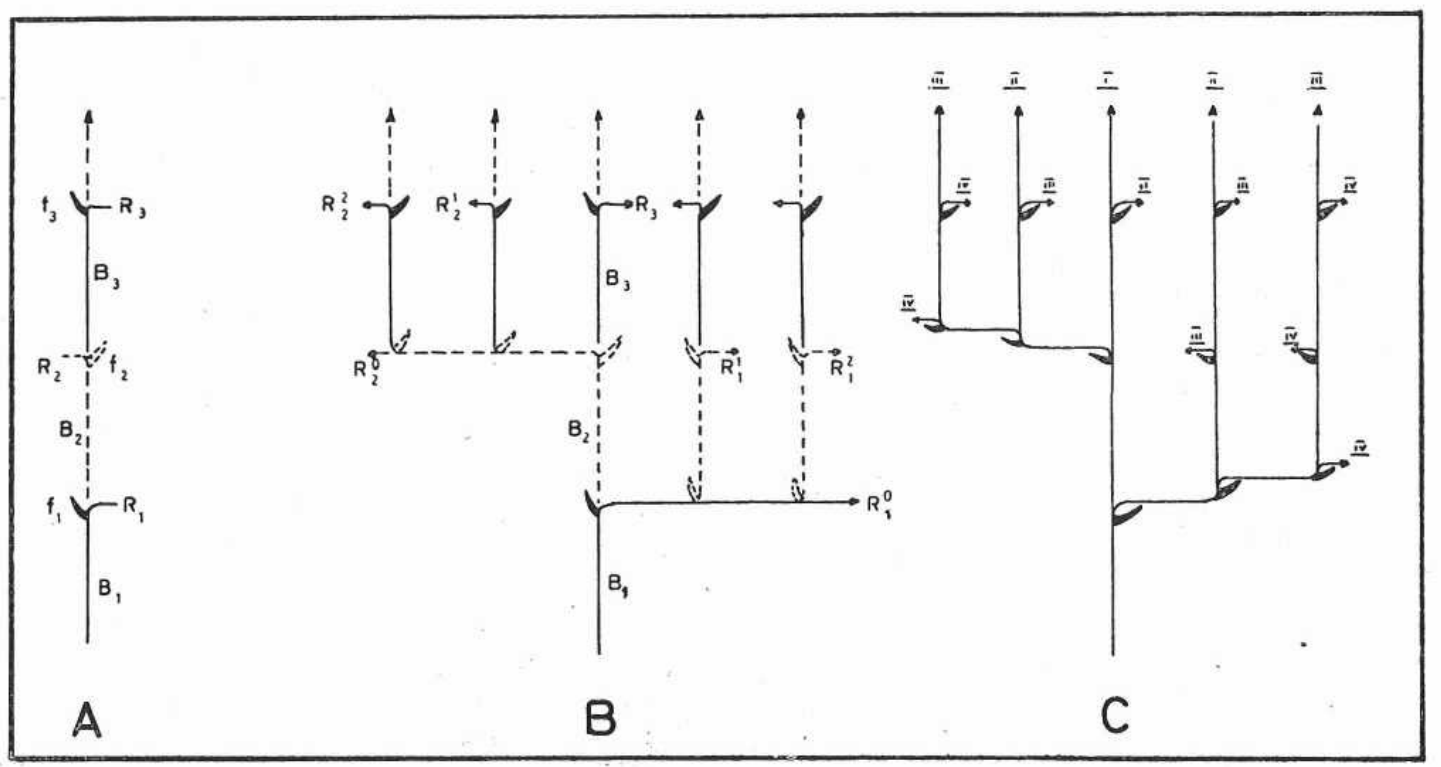




\section{Lu dans Fruits, il y a 50 ans ...}

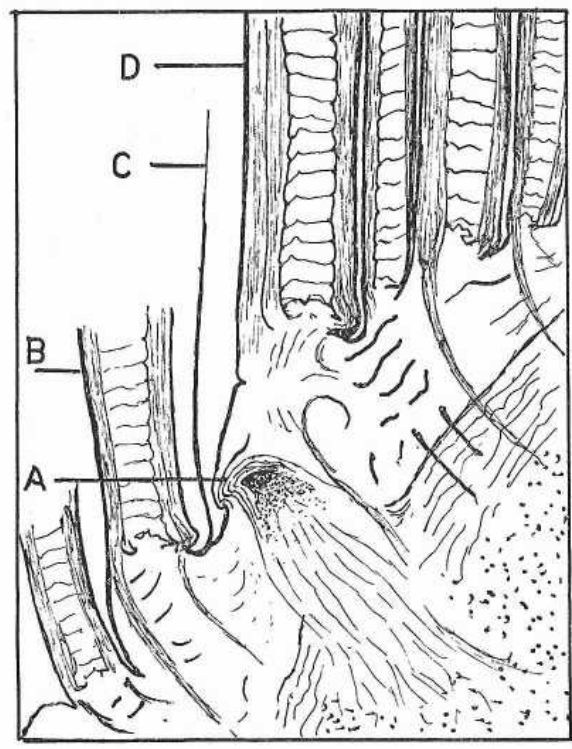

FIG. 7. - Coupe $\left(\times_{2}\right)$ d'un bourgeon avec la base des feuilles environnantes.

A. Bourgeon. - B et D. Resp. feuille inférieure et feuille supérieure, - C. Les bords de la feuille opposée.

I. Le tissu conducteur des feuilles est évidemment lié au cylindre central. Les rejets naissants ensuite projettent également leurs vaisseaux dans la couche dictyogène.

2. Dans le stade végétatif final, lors du développement des dernières feuilles, le pouvoir rejetonnant se perd. Les rares cas tératologiques que nous avons pu observer sur certaines inflorescences nous montraient un dédoublement s'effectuant directement après l'émission d'un glomérule normal, et dans le même plan que ce dernier. Une structure sympodiale entraînerait le développement en axe floral du glomérule même.

3. Il est normal que la tige centrale ne se ramifie pas au début de son développement.

4. Le schéma complet du rejetonnage monopodial ressemble à la ramification normale.

5. L'homogénéité de la famille des Musacées est conservée, tous ses membres ayant une structure monopodiale.

Cependant, une grande difficulté subsiste: la position du bourgeon. Ce dernier n'étant structuralement pas lié à la feuille opposée (fig. 7), il est logique d'admettre sa position axillaire par rapport à la feuille $B$. Mais il n'y occupe pas le centre de l'aisselle, il est dépl ıcé d'une vingtaine de degrés vers la droite, pour se loger dans l'ouverture en " $V$ " laissée par les deux bords de la feuille opposée à sa base (fig. Io).

Ce déplacement peut trouver son origine dans le niveau d'implantation des feuilles. En effet, à l'endroit où le bourgeon devrait se trouver, et au même niveau, la base de la feuille $\mathrm{C}$ fait déjà partie de la couche corticale du tubercule, et un rejet ne pourrait jamais s'y frayer un chemin vers l'extérieur.

La position actuelle du bourgeon lui permet de se développer entre les deux bords de la feuille $\mathrm{C}$, et de pousser au-dessus de la zone corticale de la feuille B, au fur et à mesure que la gaine de cette dernière se décompose. Généralement la gaine, ou ce qu'il en reste, n'oppose aucune résistance à la sortie du rejet.

Le déplacement de ro à 20 degrés que subiraient systématiquement tous les bourgeons, n'est pas visible dans leur tissu conducteur; mais nous sommes obligé de supposer que le système rejetonnant se serait adapté à cette situation. Dans cet ordre d'idée, il ne faut pas perdre de vue que chez les Strelitzoideae, les bourgeons axillaires se placent automatiquement entre les bords de la feuille opposée, puisque l'angle de divergence est égal à I/2. La genèse du phylum "Musoïdeae " où l'angle de divergence a diminué, aurait entraîné dans cette évolution les primordiums des rejets futurs, trouvant ainsi une solution mécanique à la difficulté de l'émergence de ces deniers.

\section{Remarque d'importance pratique.}

Dans une plantation de bananiers, il est malaisé de dénommer les différents pseudo-troncs qui font parfois partie d'un seul et même " pied ». On parle de rejet

Fig. 8. - Coupe $(\times 50)$ d'un primordium et des plus jeunes feuilles. Aucune trace d'autres primordiums. Les bourgeons se formeront beaucoup plus tard.

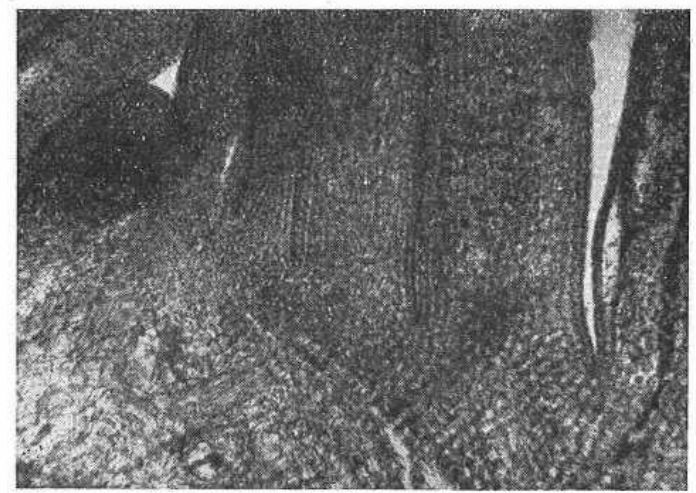




\section{Lu dans Fruits, il y a 50 ans ...}

Fıg. ro. - Position des bourgeons chez un rejeton bien développé. A. Feuille opposée au bourgeon $n^{\circ} \mathrm{I}_{4}-\mathrm{B}$. Feuille inférieure et correspondante au bourgeon $n^{0} \mathrm{I}_{4}$. - - a) tissu cortical et donc persistant de la feuille A. $b$ ) partie aérienne et caduque de la feuille A. - 1. groupe de 4 racines situées comme des bourgeons.

adulte, porteur de régime, bananier adulte, pseudotronc, fils, petit-fils, etc...

Tout matériel planté, que ce soit des rejets, des " rhizomes ", des " morceaux de rhizome ", ou des yeux, tous ces éléments donnent immanquablement lieu à un (rarement deux) pseudo-troncs conduits vers la production et le rejetonnage. Or, ces pseudo-troncs sont des véritables branches transplantées. Et les soidisant rhizomes, complexes d'un axe central, de ses ramifications et des parties persistantes des feuilles sont en réalité des tubercules.

Nous proposons, par conséquence, les termes suivants pour dénommer les différents stades et éléments $\mathrm{du}$ bananier :

- bouture : tout matériel de plantation végétatif. La nature de cette bouture sera spécifiée par des termes généralement adaptés tels que :

tubercule ou souche $=$ le tubercule adulte entier avec plusieurs bourgeons développés; éclat (de souche) - morceau d'un tubercule; rejeton — rejet de 20 à $30 \mathrm{~cm}$ dont les feuilles ne sont que des écailles ; épée $=$ rejet épée $=$ rejet atteignant $\mathrm{I} \mathrm{m}$, et dont les feuilles n'ont que des limbes lancéolés; (le terme drageon est certainement à proscrire) ;

- branche primaire : le ou les pseudo-troncs sortis du matériel de plantation. Lorsqu'on a planté un tubercule entier, les 2,3 ou 4 rejets retenus ne tardent pas à se détacher au cours de leur développement. Ils se comportent comme le produit de plusieurs éclats qu'on aurait plantés ensemble. La branche primaire pourrait aussi être appelée : axe central. Cependant, les conduites modernes tendent à ne conserver que les rejets axiaux; la ramification ne se fera pas en tous sens (comme il est le cas dans des conditions naturelles) mais dans la seule direction des rejets axiaux et la branche primaire n'occupera jamais une position centrale. Le terme " axe cen-

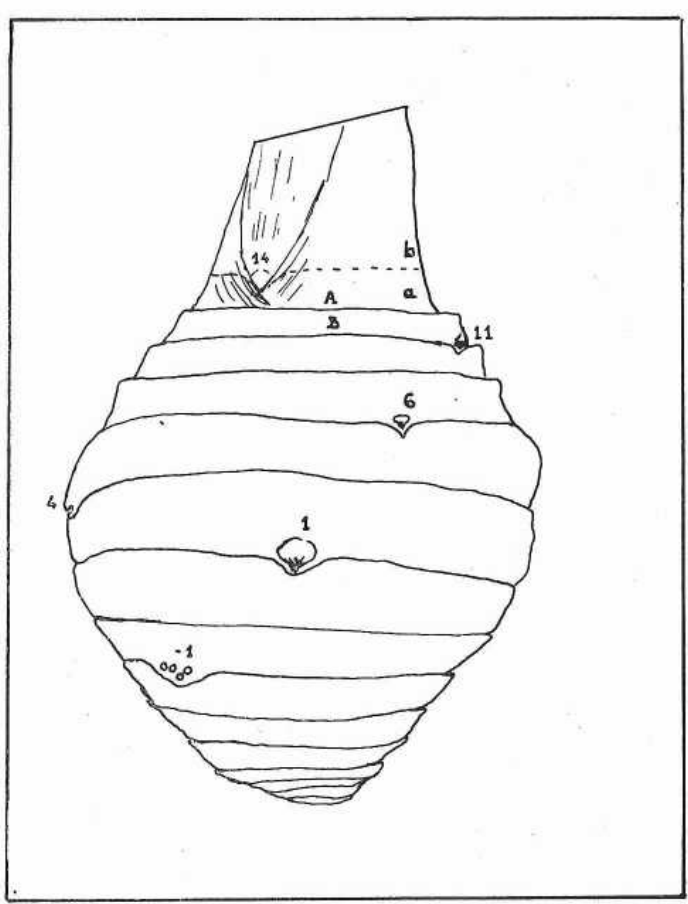

tral » devrait cependant être employé dans des considérations théoriques.

- rejeton : la toute jeune branche secondaire de 20$30 \mathrm{~cm}$ avec des écailles comme feuilles.

- épée ou ramean : rejet épée $=$ sword sucker $=$ (drageon) : la branche produit des feuilles au limbe lancéolé.

- branche secondaire ou "branche " tout court = rejet adulte : les feuilles portent des limbes normaux.

A la rigueur, et pour des études plus précises, on pourra parler de " branches du second degré, du troisième degré, " etc... désignant ainsi les rejets dénommés parfois fils, petit-fils, etc...

- bananier (ou pied de bananier) : tout le complexe issu del'élément de plantation (y compris les branches primaires, secondaires, etc...).

\section{LE SYSTĒME REJETONNANT}

\section{A. Diagramme.}

Chaque rejet correspond à une feuille, quelle soit opposée ou aposée. Le rejetonnage suit donc littérale- ment la phyllotaxie. Les rejets utiles, ceux qui deviennent des branches, ont pris naissance à un stade très jeune de la branche qui les porte. A ce stade, l'angle de divergence entre deux feuilles n'a pas encore 


\section{Lu dans Fruits, il y a 50 ans ...}

dépassé I40 degrés (fig. 5). Comme il est montré dans le diagramme du rejetonnage (fig. 9), les rejets prennent pratiquement naissance selon un pentagone irrégulier formé d'abord par les points $I, 2,3,4,5$. Ce pentagone tourne ensuite vers la droite, par pas de 20 degrés environ. Avec l'évolution de l'angle de divergence chez les feuilles, le pentagone évolue vers un tétragone de plus en plus aplati ; mais ce stade ne nous intéresse plus, puisque ces rejets ne se développent presque jamais.

Le premier véritable rejet se forme à un stade déjà avancé du rejeton. Nous avons constaté le fait remarquable que les feuilles antérieures semblent être accompagnées, non pas de bourgeons, mais de racines. Il est connu qu'une grande partie des racines du bananier naissent par groupes de quatre. Or, on remarque par exemple dans la figure ro que ces groupes de quatre racines sortent aux mêmes endroits que les rejets, c'est-à-dire exactement là où les deux bords d'une gaine foliaire se sont soudés à la base. Nous faisons cependant remarquer que ces groupes n'occupent pas tous les endroits disponibles, et que, par conséquent, plusieurs feuilles ne sont accompagnées ni par des racines, ni par des bourgeons. Ajoutons que d'autres racines isolées émergent en des endroits quelconques.

Somme toute, il nous semble que pendant un certain temps après l'émergence, des primordia secondaires donnent lieu à des racines groupées par quatre : ensuite, au stade " épée ", ces primordia (sous quelle influence ?) développent des bourgeons, dont certains deviennent des rejets.

\section{B. Evolution du rejetonnage.}

Tous les bourgeons ne deviennent pas des rejets. On doit admettre avec Brouhns (I957) et SubrA, GuILLEMOT (I96I) que le premier nouveau bourgeon sera presque toujours situé à l'opposé du point d'attache du rejet avec le " pied-mère " (nous préférons le terme : branche-mère). En faisant démarrer le diagramme de la figure 9 par ce bourgeon ( $\left.n^{0} \mathrm{I}\right)$, il devient clair que les bourgeons 2 et 5 , coincés dans l'angle formé par la branche-mère et le rejet ne se développeront pas davantage. Si jamais ils y parviennent, par exemple après l'élimination de la branche-mère (suite à la récolte), ces rejets seront neuf fois sur dix très faibles et de valeur nulle. Le rejetonnage utile va donc se concentrer sur les bourgeons I, 3, 4 et leurs suivants. On a remarqué à Yangambi ainsi qu'à Mulungu (altitude) que les bourgeons 6 et II ne deviennent généralement pas des rejets, mais bien les bourgeons 3,4 ou 8, 9 (fig. 9). Lorsque le rejet 6 par exemple parvient à se développer, il sera généralement handicapé par le rejet $I$. Cependant, il existe des cas où les deux rejets $I$ et 6 (ou les rejets 3 et. 8 , ou encore 4 et 9) se développent simultanément. Lorsque donc on remarque sur un pied deux rejets fort rapprochés et d'une vigueur égale, il faudra éliminer systématiquement celui qui se présente à votre gauche. En effet, ce sera très probablement le plus vieux des deux; et donc il se trouve vis-à-vis de l'autre en état de sous-développement.

Quoiqu'on puisse affirmer avec les auteurs précités que seul le rejet axial est à conserver, et que tous les autres, formés à un niveau plus élevé et donc mọins bien assis dans le sol, sont à éliminer, il faut inciter le cultivateur à la prudence. Chez plusieurs variétés, il s'est révélé que le rejet peut se trouver handicapé dans son développement par des élements encore insuffisamment connus (la compacité locale de la terre ? une stérilité fort localisée autour du rejet ?). Il sera donc prudent d'observer l'évolution de quelques autres premiers rejets (par exemple 3,4 ). Si, au stade épée, ils s'avèrent finalement plus vigoureux que le $\mathrm{n}^{\circ} \mathrm{I}$, on aura tous les avantages à éliminer ce dernier et à conserver un des autres. Cette méthode sera particulièrement à conseiller pour les plantains.

\section{L'individualisation du rejet.}

L'émission des écailles foliaires se fait grâce aux substances venant du rejet-mère par la zone d'étranglement, là où le bourgeon avait pris naissance. Cette zone s'élargit aussi longtemps que le nouveau rejet vit aux dépens de la branche-mère. Entre-temps, le système radiculaire ainsi que le nouveau tubercule se développent lentement, recevant eux aussi les matières nécessaires de la branche-mère. Il n'a pas encore été établi sous l'influence de quels facteurs les feuilles se mettent à développer un limbe. Ce moment n'est par exemple pas défini par un certain potentiel qui aurait été entassé dans le nouveau tubercule, puisque déjà, au début de leur développement, des rejets peuvent émettre des feuilles larges, quoique d'une dimension réduite : ce seront les "water-suckers ", les rejets faibles, qui ne parviendront même jamais à former un tubercule convenable.

En tout cas, le métabolisme du rejet subit une transformation à ce stade, et, dans des conditions normales, le nouveau rejet va se développer sans aide de la branchemère, les limbes permettant dorénavant la photosynthèse. Le rejet s'est individualisé. Bien que la zone d'étranglement ne disparaisse pas toujours, sa valeur devient quasi nulle. A Yangambi, dans un climat chaud et humide, les tubercules-mères pourrissent 
régulièrement après la récolte, mais la pourriture ne se communique que rarement aux rejets bien développés. Le réseau vasculaire du rejet s'est donc isolé.

Les rejetons ont par contre été contaminés lorsque le tubercule coupé était lui-même encore jeune, et que les rejets ne s'étaient donc pas encore individualisés. Dans les pépinières de Yangambi où on assurait la multiplication végétative du plantain, un des traite- ments consistait en un recépage des pieds (épées) plantés depuis quatre mois. : les tubercules étant trop jeunes, la pourriture s'y était vite déclarée et les rejetons développés autour d'eux furent tous contaminés.

Inversement, en recépant un rejet individuel, qu'on reconnaît facilement aux feuilles limbées, le rejet-mère n'en subira aucune influence. Il n'en est pas de même lors du recépage des rejetons. Dans un essai (B. E. 233)

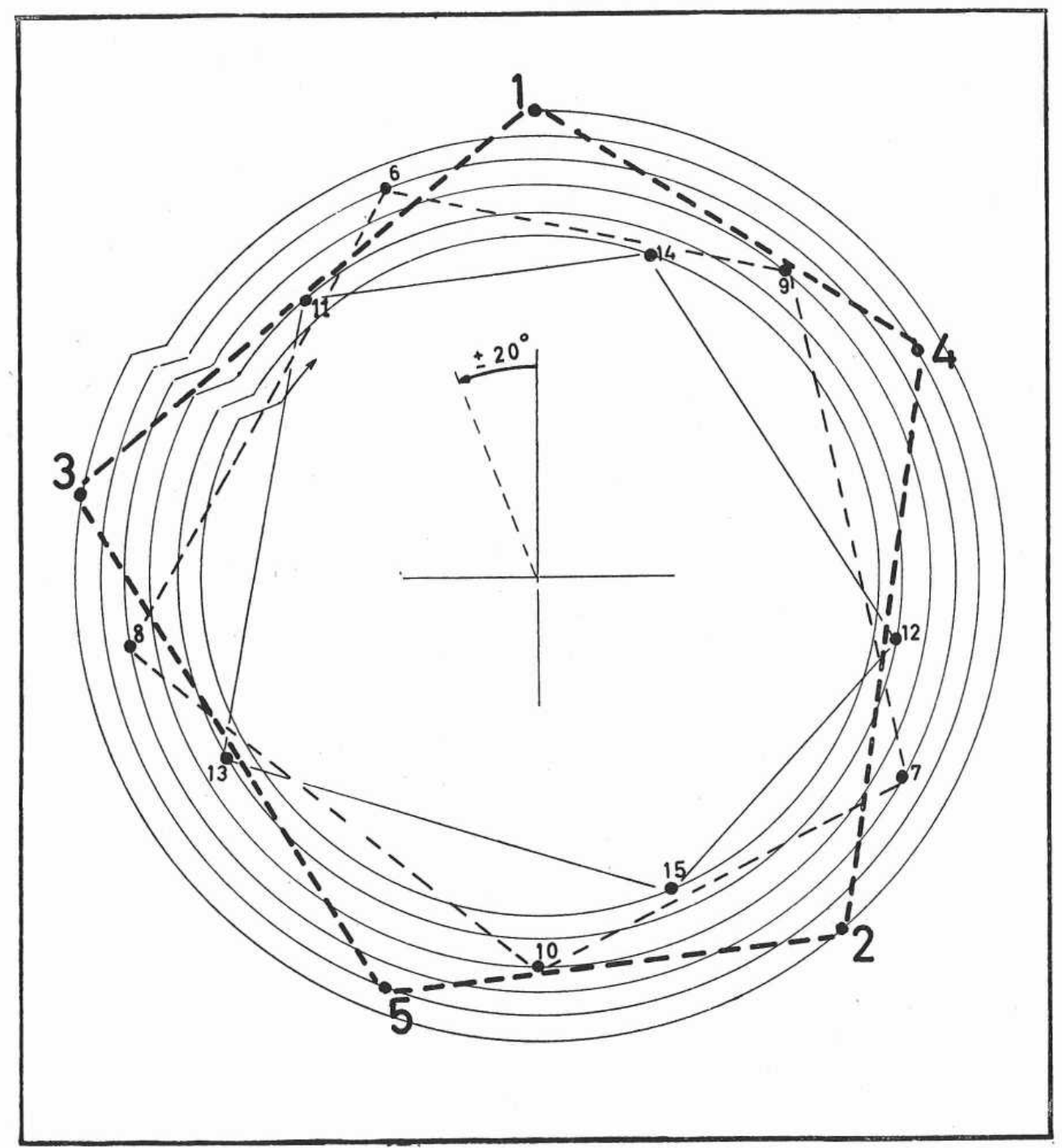

Fig. 9. - Diagramme du rejetonnage. 


\section{Lu dans Fruits, il y a 50 ans ...}

établi à Yangambi depuis I957, on comparait e. a. la conduite classique (recépage de tous les rejets sauf le plus vigoureux) à une conduite naturelle qui laissait se développer tous les rejets, sauf les " water-suckers ». Or, le bananier traité était le Bosua, plantain géant. Dans les conditions de Yangambi, le tubercule ne produit que des rejetons; rares sont les rejets ipées, et les rejets individuels sont vraiment exceptionnels. Ce n'est qu'à la floraison de la branche-mère que les rejetons - tous à la fois - continuent leur développement. Afin de stimuler le rejeton principal, le recépage systématique des autres rejetons fut donc envisagé. Les résultats furent étonnant (INEAC, I959) : non seulement le rejeton principal ne fut pas influencé favorablement, mais en plus le rendement du pied-mère se ressentait du traitement. Bien que l'infériorité de la conduite classique sur le Bosua ne fut pas significative au point de vue rendement, sa fréquence atteignait les Ioo $\% !$ La coupe des rejetons influence donc le tuberculemère, via la zone d'étranglement.

Pour les raisons exposées dans ces derniers paragraphes, l'élimination d'un rejet ne pourra donc se faire que lorsqu'il aura atteint le stade " épée ", c'està-dire lorsque les feuilles commencent à former un limbe lancéiforme. A ce moment, la zone d'étranglement connaît peut-être encore une certaine activité, mais attendre plus longtemps risque d'être nuisible au rejet à conserver, concurrencé de plus en plus par d'autres rejets.

\section{Application et exploitation du concept " reje- tonnage en pentagone $"$.}

I. L'établissement de la phyllotaxie du bananier nous a permis de comprendre le système du rejetonnage. Les rejets émergent pratiquement en des pentagones irréguliers, qui se superposent en tournant vers la droite avec un angle de divergence d'à peu près 20 degrés. Seul le premier de ces pentagones semble important et tout particulièrement son premier rejet, le rejet axial, celui-ci assurant généralement la relève.

2. Lorsque ce rejet ne se développe pas, l'on fera appel à un des deux rejets 3 ou 4 du pentagone basal ; il est clairement établi que les rejets 2 et 5 ne se prêtent pas à une conduite rationnelle ; rares sont les cas d'ailleurs où ils se développent réellement.

3. Certaines plantations commencent à une densité faible et conservent au cours de la taille deux rejets par bananier. Lesquels faut-il retenir ? Théoriquement les rejets 3 et 4 seraient préférables, étant donné leur distance par rapport au centre. L'élimination du rejet axial vigoureux ne serait pourtant pas logique. Il fau- drait donc se concentrer sur les rejets I et 3 , leur angle de divergence atteignant presque 90 degrés. Cependant, ces rejets se suivent de trop près, et, dans des sols moyens, ils vont plutôt se concurrencer. Dans ce cas, il sera opportun de retenir, à côté du rejet $I$, un rejet du second pentagone, en l'occurrence le numéro 7 ou 8 . Cette technique se réalisera en deux étapes :

a. Des trois premiers rejets sortis $(\mathrm{I}, 3,4)$ seul le rejet axial sera conservé (nous présupposons ici l'atrophie normale des bourgeons 2 et 5 ).

b. On attendra l'émission d'un nouveau cercle de rejets, pour retenir le numéro 7 ou 8 , selon la position pratique (le plus éloigné du rejet axial).

4. L'étude du pouvoir rejetonnant chez des variétés différentes devient beaucoup plus rationnelle en appliquant l'idée du pentagone.

Beaucoup de variétés (Cavendish, Argent, Pomme) semblent posséder un pouvoir auto-conducteur, en ce sens que les bananiers ne développent que quelques rejets (2 à 4) sans l'intervention d'une taille. Ces rejets apparaissent au-dessus du sol simultanément ou à des époques différentes. Ils peuvent se concurrencer dans le premier cas; ils connaitront un développement équilibré dans le second cas. Par contre les plantains en général laissent se développer tous leurs bourgeons. Dans pareilles circonstances, et pour des bananiers poussant dans des conditions moyennes, toutes les nouvelles branches tardent au stade rejeton aussi longtemps que la branche-mère n'a pas fleuri. Ensuite, elles pousseront toutes à la fois en se faisant concurrence et la taille devient ici extrêmement difficile.

Il y a donc lieu de caractériser les variétés par leur pouvoir de donner des rejets utiles, ce qui revient à suivre systématiquement, et de près, le processus du rejetonnage.

Pour observer si, à côté du rejet axial, les autres rejets développés sortent à égale hauteur du tubercule, ou bien s'ils dérivent de bourgeons bien postérieurs, il ne sera pas nécessaire de déterrer le tout. Si l'angle formé par ces rejets respectifs et le rejet axial est légèrement inférieur à 90 degrés (par exemple 3 et 4 ) ils appartiennent au pentagone de ce dernier ; si cet angle dépasse nettement 90 degrés (avec 7 et 8 ) les rejets font partie du pentagone suivant. Quant au rejet 9, l'angle de divergence sera de l'ordre de 40 degrés, et ce rapprochement (impossible pour le rejet 4) permet de le reconnaître aisément; de plus, la grande différence d'âge (I à 9 !) se matérialise par une position plus élevée et plus rapprochée du centre vis-à-vis du rejet axial. 


\section{Lu dans Fruits, il y a 50 ans ...}

Reste la position des rejets 6 et II. Comme nous l'avons fait remarquer précédemment ( $\left.\S_{3} \mathrm{~B}\right)$, le rejet n 6 ne se développe convenablement qu'en cas d'atrophie ou de sous-développement du rejet axial. Le rejet $\mathrm{n}^{\circ}$ II occupe une position comparable à celle du rejet $n^{\circ} 9$ mais à droite du rejet axial; il ne se développe que rarement (du moins chez les plantains).

Le tout consiste donc en un examen progressif où les positions supposées se confirment ou s'infirment avec l'observation des autres rejets.

Une fois connus les pentagones auxquels appartiennent les rejets, il est facile d'en déduire l'histoire de leur développement. Voici les cas qui peuvent se présenter et leurs conséquences.

A. Les rejets se développent simultanément (intervalles $<3$ semaines).

I. Ils appartiennent au même pentagone : la variété ne possède pas de pouvoir auto-conducteur, elle fournira normalement une foule de rejetons mais pas de rejets utiles.

2. Ils appartiennent à des pentagones différents : dans chaque pentagone, quelques primordiums se sont atrophiés et seuls, un ou deux bourgeons se sont développés. La variété possède un certain pouvoir auto-conducteur.

B. Les rejets se développent successivement (intervalles $>$ I mois).

I. Ils appartiennent au même pentagone : certains bourgeons du pentagone se sont atrophiés (ou n'ont même pas pu se développer), d'autres ont connu une période latente.

2. Ils appartiennent à des pentagones différents : nous nous rapprochons ici du cas A I, avec la différence qu'une partie des rejets manquent par pentagone.

Une étude systématique de toutes ces éventualités avait été entamée sur toutes les variétés de la collection de bananiers à Yangambi ( 54 plantains, 25 autres variétés). Les événements politiques en I960 nous ont empêché de tirer des conclusions substantielles. Tous les plantains présentent certainєment le cas A I, avec une légère tendance vers le cas B 2 .

\section{CONCLUSION}

Pendant la première partie de sa vie végétative, le bananier émet un nombre variable de rejets, dont chacun correspond dans l'espace à une feuille. Le plan du rejetonnage est donc la copie de la phyllotaxie. Puisque la période de rejetonnage qui nous intéresse c'est-à-dire celle qui produit les futures nouvelles branches - correspond au stade où les feuilles se succèdent avec un angle de divergence compris entre 135 et I45 degrés environ, des groupes de cinq rejets successifs formeront en théorie des pentagones irréguliers. Ces pentagones se superposent en tournant légèrement vers la droite (environ 20 degrés).

En pratique, les rejets ne se développent pas tous.
Selon que les rejets se développent simultanément ou successivement, en groupes entiers ou partiels, le système rejetonnant présentera une grande variation, dont chaque cas peut caractériser une variété.

Enfin, l'emploi du " système pentagonal " est très utile dans les investigations sur le rejetonnage et la conduite des bananiers.

Dans le domaine morphologique, et suite à notre étude du bourgeonnement, il nous paraît à peu près certain que le bananier est bâti comme un monopodium, où les rejets sont des véritables branches, qui prennent naissance dans l'aisselle des feuilles sousjacentes.

\section{BIBLIOGRAPHIF}

Barker, W. G. - "A System of Maximum Multiplication of the Banana Plant ». Trop. A griculture, I959, $\mathrm{n}^{\circ} 4$.

Brounns, G. - " Note sur la Croissance du bananier [Gros-Michel] ". Fruits, 1957, vol. 12, n ${ }^{\circ} 6$.

Dusras, J. - * Contribution à l'étude du développement du Bananier nain \%. Fruits, 1955, vol. 10, n० 8 .

I. N. E. A. C. - Rapport Annuel 1959. Centre de Recherche Yangambi. Division des Plantes Vivrières.

Kervegant. - "Le Bananier et son exploitation ", Paris, 1935.

Muller, J. - "Amélioration du bananier indigène ». C. $R$, de la Semaine Agricole de Yangambi, 1947, Communication $\mathrm{n}^{\circ} 2 \mathrm{r}$.

Schumann, K. - " Musaceae » in Engler, Pfianzenr., IV, 45 Hl, 45 p., Io fig. (I900)
Simmonds. - "Bananas \#, Longmans, I959.

Simmonds, N. W. and Shepherd, K. - "The taxonomy and origins of the cultivated bananas $»$ in the Jul. of the Linnean Soc. of London, vol. LV, n 359 , I955.

Sкuтсн, - "Anatomy of leaf of banana, Musa sapiemtum L, var. hort. Gros Michel ». Bot. Gazette, LXXXIV, 1927.

SкUтсн. - «Anatomy of the axis of the banana ", Bot. Gazette, XCIII, I932.

Subra et Guillemot, J. - "Contribution à l'étude dı rhizome et des rejets du bananier». Fruits, I96r, n० $\mathrm{n}^{\circ}$, p. 19.

Walker, A. - «Le Bananier Plantain au Gabon». Rev. de Bot. Appl. et d'Agric., 1931, $\mathrm{n}^{\circ} \mathrm{II} 3$. 